\title{
La influencia de los productos hidrofugantes en la succión del ladrillo cerámico cara vista
}

\section{J. A. CORONADO MARTíN, A. GARCía SANTOS, J. F. PADIAL MOLINA}

E.T.S. de Arquitectura, Univ. Politécnica de Madrid, Av. Juan de Herrera 4, 28040, Madrid, España.

\begin{abstract}
Se analiza la variación de la tasa de absorción inicial y del nivel de agua superficial que sufre la cerámica porosa cara vista protegida por la incorporación de un tratamiento con productos hidrofugantes.

Se selecciona la cerámica en relación con su proceso de fabricación: manual, prensado y extrusionado. Los productos hidrofugantes se seleccionan en función de su composición química en seis tipos clasificados en monocomponente y bicomponente.

La metodología empleada está basada en la norma UNE-EN 772-11:2001 “Tasa de absorción de agua inicial”, observándose que todos los productos hidrofugantes reducen los parámetros de tasa de absorción inicial y nivel de agua superficial de la cerámica porosa cara vista, respecto de la cerámica sin hidrofugar. La variación de dichos parámetros está determinada por el tipo de hidrofugante, reduciendo en todos los casos la tasa de absorción inicial más de un 84,37\% y el nivel de agua superficial en más de un 52,55\%.

Según los resultados obtenidos, se recomienda la elección del hidrofugante H1 de componente siliconatos, ya que, en general, es el producto que produce la mayor reducción de la tasa de absorción y del nivel de agua superficial en todos los tipos de cerámica ensayados.
\end{abstract}

\section{INTRODUCCIÓN}

La investigación analiza la variación de los parámetros de tasa de absorción inicial y nivel de agua superficial que sufre la cerámica porosa cara vista al ser tratada superficialmente con productos hidrofugantes, clasificados en función de su composición, para poder identificar qué producto hidrofugante es el que produce la mayor reducción de los parámetros en cada uno de los tipos de cerámica estudiada.

Se identifican el mínimo número de tipos básicos de cerámica en relación con su proceso de fabricación: manual, prensado y extrusionado. Se selecciona un modelo de cada tipo de cerámica, incluyendo dos tipos de cerámica extrusionada, con diferentes valores del coeficiente de absorción.

Los productos hidrofugantes son una impregnación, que penetra a través de la porosidad del material sobre el que se aplica reduciendo drásticamente la succión de agua, sin taponar los poros, y por tanto, sin alterar significativamente la permeabilidad del soporte al vapor de agua. La adherencia entre una impregnación y la cerámica se debe a varios factores pero fundamentalmente a la diferencia de tensiones superficiales existentes entre ambos materiales. La composición de los hidrofugantes está basada en la utilización de materiales poliméricos que poseen una menor tensión superficial que el resto de los materiales utilizados en construcción y de esta manera garantizan su unión (1).

Cada producto hidrofugante seleccionado es aplicado a cada modelo de cerámica porosa seleccionada, cuantificándose la variación de la tasa de absorción inicial y del nivel de agua superficial que produce cada producto y así poder determinar el hidrofugante que mayor variación produce en cada tipo de cerámica seleccionada.

\section{MATERIALES}

\subsection{Cerámica porosa cara vista}

En relación con los tipos de fabricación: manual, prensado y extrusionado, se realiza la selección del material cerámico, tomando un modelo de cada tipo de cerámica. Del tipo de fabricación extrusionada se seleccionan tres modelos en base al valor de su coeficiente de absorción.

Se caracteriza a todas las cerámicas seleccionadas, calculando su coeficiente de absorción, según la norma UNE 67.027-84, obteniendo los valores indicados en la tabla I, según el proceso de fabricación (2).

TABla I. Valores del COeficiente de abSORCión (CA) DE LA CERÁMicA SELECCIONADA.

\begin{tabular}{|c|c|c|c|c|}
\cline { 2 - 5 } \multicolumn{1}{c|}{} & \multicolumn{3}{c|}{ Tipo de fabricación de la cerámica } \\
\cline { 2 - 5 } & Manual & Prensado & \multicolumn{2}{c|}{ Extrusionado } \\
\cline { 2 - 5 } & Cerámica 1 & Cerámica 2 & Cerámica 3 & Cerámica 4 \\
\hline $\begin{array}{c}\text { Coeficiente } \\
\text { absorción } \\
\text { CA (\%) }\end{array}$ & 20,08 & 7,43 & 12,08 & 6,25 \\
\hline
\end{tabular}




\subsection{Productos hidrofugantes}

Los productos hidrofugantes para esta investigación se estructuran en dos grandes grupos: orgánicos y órganosilícicos que se subdividen en siliconatos y en los derivados de la silicona: silanos, siloxanos y resinas de silicona (3). Los estudiados en este artículo son los órgano-silícicos ya que en la actualidad son los más utilizados para la aplicación sobre materiales cerámicos porosos cara vista y se pueden clasificar en: mono-componente y bi-componente (4).

- Mono-componente: se obtienen a partir de un alquiltriclorosilano y son los siguientes:

- Siliconatos

- Silanos

- Siloxanos

- Alquilalcoxisilanos oligoméricos

- Alquilalcoxisilanos poliméricos

- Resinas

- Bi-componente: se obtienen a partir de la mezcla de dos productos mono-componente.

Los productos hidrofugantes órgano-silícicos son una impregnación que penetra a través de la porosidad del material sobre el que se aplica. Los hidrofugantes son tratamientos que forman una película continua que recubre la superficie de los poros y que reducen drásticamente la succión de agua, sin taponar los poros, y por tanto, sin alterar significativamente la permeabilidad del soporte al vapor de agua (3)(5). Esta investigación pretende cuantificar la variación de la tasa de absorción inicial y del nivel de agua superficial que producen los hidrofugantes en el soporte cerámico.

La selección de los productos hidrofugantes se realiza en función de su composición (2) (4) (6) (7).

\section{METODOLOGÍA}

La metodología empleada está basada en la norma UNEEN 772-11:2001 “Tasa de absorción de agua inicial” (8).

De cada modelo de cerámica seleccionada, se escogen 7 muestras que es hidrofugada en su cara de testa mediante pincel hasta saturación del soporte, según indicaciones de los fabricantes. El tiempo de secado de las muestras hidrofugadas es de 24 horas (el tiempo máximo recomendado por los fabricantes para que el hidrofugante esté completamente seco), realizando el ensayo entre las 24 y las 72 horas después de hidrofugarlas.
Una vez hidrofugada la cerámica, se introduce su cara de testa en agua, quedando la cerámica 2,5 mm sumergida, manteniendo la cerámica sumergida 1 minuto, se pesa y se coloca en la mesa de reproducción donde se realiza una fotografía de la cara de canto (2)(6)(7)(9)(10). Se introduce de nuevo en la cubeta y se repite el proceso para los tiempos 2, $5,10,15,20,25$ y 30 minutos. Se obtiene la tasa de absorción inicial en cada uno de los tiempos mediante la siguiente formula:

$$
\mathrm{C}=(\mathrm{Mi}-\mathrm{Ma}) /(\text { As } \times \mathrm{t})
$$

Siendo C la tasa de absorción, Mi la masa de la probeta después de inmersión, Ma la masa de la probeta a temperatura ambiente, As la superficie bruta de la cara sumergida y $\mathbf{t}$ el tiempo.

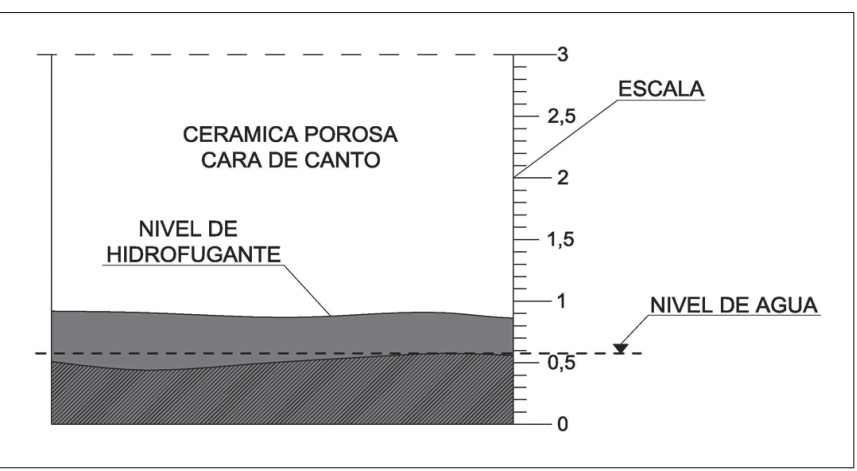

Figura 1. Esquema de obtención de nivel de agua.

El aumento de nivel de agua en la superficie de la cerámica se realiza analizando la fotografía obtenida mediante un programa informático. La succión en la probeta de ladrillo cerámico, tanto del producto hidrofugante como del agua, se produce de manera no uniforme (como se representa en la figura 1), ya que el material es poroso y por lo tanto, no homogéneo. Para la obtención del nivel de agua, se marca el máximo nivel dejado en la superficie de la probeta trasladándolo a la escala para obtener su valor (2).

\section{RESULTADO DE LOS ENSAYOS}

Los resultados obtenidos en el ensayo son gráficas obtenidas de cada tipo de cerámica hidrofugada con cada uno de los productos hidrofugantes seleccionados, de la tasa de absorción y del nivel de agua superficial.

En la figura 2 se muestra como ejemplo, la gráfica de la tasa de absorción inicial obtenida para las cerámica 2.

TABLA II. SELECCIÓN Y CARACTERÍSTICAS DE LOS PRODUCTOS HIDROFUGANTES.

\begin{tabular}{|c|c|c|c|c|}
\hline Clasificación & Hidrofugante & Componente & Solución & Densidad (Kg/litro) \\
\hline \multirow{5}{*}{ Mono-componente } & H1 & Siliconatos & Acuosa & 1,001 \\
\hline & $\mathrm{H} 2$ & Silanos & Disolvente (xileno) & 0,8 \\
\hline & H3 & Siloxanos Oligoméricos & Disolvente (white spirit*) & 0,9 \\
\hline & H4 & Siloxanos Poliméricos & Disolvente (white spirit*) & 0,83 \\
\hline & H5 & Resina Fluorada & Acuosa & $0,95-1,00$ \\
\hline Bi-componente & H6 & Silanos/Siloxanos & Acuosa & 1 \\
\hline
\end{tabular}

* White Spirit: Hidrocarburo alifático derivado del petróleo. 


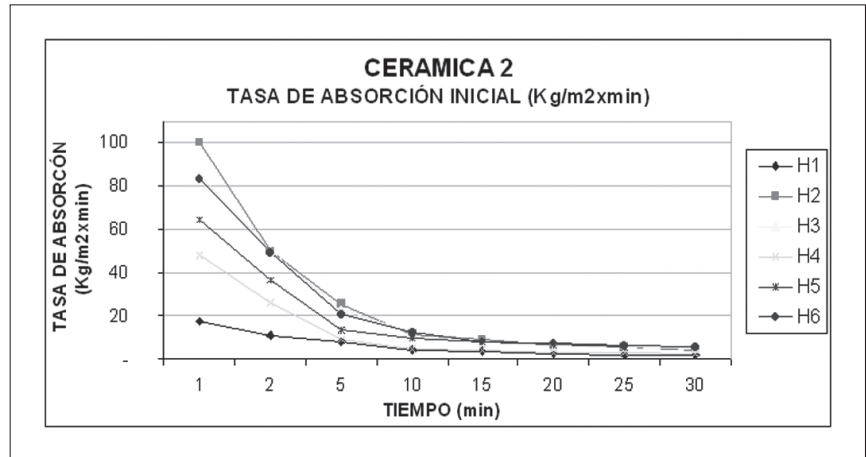

Figura 2. Tasa de absorción inicial de las cerámica 2 hidrofugada con cada tipo de producto.

No se representa los valores de la tasa de absorción inicial de la cerámica 2 sin hidrofugar (H0), ya que se desvirtuarían las gráficas por su elevado valor.

En la figura 3 se muestran como ejemplo, la gráfica del nivel de agua superficial obtenida para la cerámica 3 .

No se representan los valores de la cerámicas 3 sin hidrofugar (H0) del nivel de agua superficial, ya que se desvirtuarían las gráficas por su elevado valor.

\section{ANALISIS E INTERPRETACION DE RESULTADOS}

Todos los hidrofugantes producen una modificación en los parámetros de tasa de absorción y en el nivel de agua superficial en todos los tipos de cerámica ensayados, respecto de la cerámica sin hidrofugar. Se analizan los resultados de cada cerámica ensayada.

\section{Cerámica 1}

El hidrofugante que más reduce la tasa de absorción inicial es el $\mathrm{H} 4$ de componente siloxanos poliméricos, excepto en el minuto 1 que es el producto H6 de componente silanos/ siloxanos el que produce la mayor reducción. El nivel de agua superficial presenta valor cero con el hidrofugante H1 de componente siliconatos en todos los tiempos ensayados.

\section{Cerámica 2}

El hidrofugante que más reduce la tasa de absorción inicial es el H1 de componente siliconatos en todos los tiempos ensayados y el producto $\mathrm{H} 5$ de componente resina fluorada es que mayor reducción produce en todos los tiempos del nivel de agua superficial.

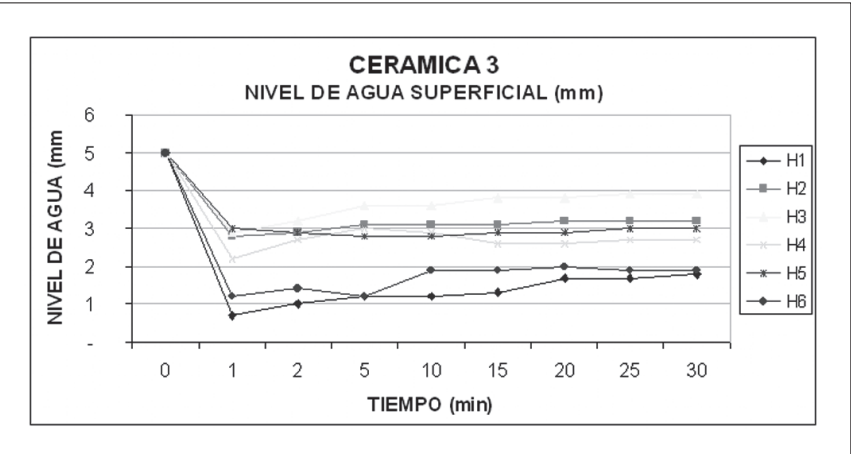

Figura 3. Nivel de agua superficial de la cerámica 3 hidrofugada con cada tipo de producto.

\section{Cerámica 3}

El hidrofugante que más reduce la tasa de absorción inicial es el $\mathrm{H} 4$ de componente siloxanos poliméricos. El nivel de agua superficial presenta mayor reducción con el hidrofugante $\mathrm{H} 1$ de componente siliconatos en todos los tiempos ensayados.

\section{Cerámica 4}

El hidrofugante que más reduce la tasa de absorción inicial es el $\mathrm{H} 1$ de componente siliconatos en todos los tiempos ensayados. El nivel de agua superficial presenta mayor reducción con el hidrofugante $\mathrm{H} 1$ de componente siliconatos y con el producto H6 de componente silanos/siloxanos con valor cero en todos los tiempos ensayados, excepto el producto H6 en el minuto 30.

\section{Tasa de absorción}

Todos los hidrofugantes reducen la tasa de absorción inicial de la cerámica respecto de la cerámica sin hidrofugar (H0). Para poder determinar el producto hidrofugante que produce mayor reducción de la tasa de absorción inicial, se cuantifica para cada tiempo ensayado, la reducción que produce cada producto en cada tiempo respecto de la cerámica sin hidrofugar (H0). Para cada tiempo se obtiene el porcentaje $(\%)$ de reducción respecto a la cerámica sin tratar. De todos los porcentajes obtenidos para cada tiempo, se obtiene el porcentaje de reducción total, aplicando la media aritmética a los porcentajes obtenidos para cada tiempo ensayado. En la tabla III se presenta el porcentaje de reducción de la tasa de absorción inicial respecto a la cerámica sin tratar, que produce cada hidrofugante en cada tipo de cerámica ensayada (signo + aumento; signo - disminución).

TABla III. PORCENTAJE DE VARIACIÓN DE LA TASA DE ABSORCIÓN INICIAL RESPECTO DE LA CERÁMICA SIN HIDROFUGAR.

\begin{tabular}{|c|c|c|c|c|c|}
\hline \multicolumn{5}{|c|}{ Tasa de absorción inicial. Porcentaje (\%) de variación respecto de la cerámica sin hidrofugar (H0) } \\
\hline Hidrofugante & H1 & H2 & H3 & H4 & H5 \\
\hline Cerámica 1 & $-98,76$ & $-99,04$ & $-97,84$ & $-99,37$ & $-97,08$ \\
\hline Cerámica 2 & $-98,61$ & $-95,45$ & $-95,65$ & $-97,76$ & $-96,39$ \\
\hline Cerámica 3 & $-96,69$ & $-94,15$ & $-94,12$ & $-97,08$ & $-94,93$ \\
\hline Cerámica 4 & $-99,21$ & $-86,61$ & $-84,37$ & $-93,78$ & $-93,76$ \\
\hline Cerámica 5 & $-98,99$ & $-95,41$ & $-93,11$ & $-94,17$ & $-94,94$ \\
\hline
\end{tabular}


TABla IV. PorCENTAJE DE VARIACIÓN DEL NIVEL DE AGUA SUPERFICIAL RESPECTO DE LA CERÁMICA SIN HIDROFUGAR.

\begin{tabular}{|c|c|c|c|c|c|c|}
\hline \multicolumn{6}{|c|}{ Nivel de agua superficial. Porcentaje (\%) de variacion respecto de la ceramica sin hidrofugar (h0) } \\
\hline Hidrofugante & H1 & H2 & H3 & H4 & H5 & H6 \\
\hline Cerámica 1 & -100 & $-96,58$ & $-94,34$ & $-97,63$ & $-94,74$ & $-96,05$ \\
\hline Cerámica 2 & $-81,07$ & $-80,01$ & $-77,96$ & $-79,22$ & $-90,48$ & $-89,57$ \\
\hline Cerámica 3 & $-94,77$ & $-88,37$ & $-86,75$ & $-89,71$ & $-88,89$ & $-93,4$ \\
\hline Cerámica 4 & -100 & $-76,9$ & $-69,93$ & $-81,87$ & $-70,08$ & $-97,9$ \\
\hline Cerámica 5 & $-85,81$ & $-65,53$ & $-65,47$ & $-52,55$ & -65 & $-90,93$ \\
\hline
\end{tabular}

Se observa en la tabla III que es el hidrofugante H1 de componente siliconatos es el que produce mayor reducción de la tasa de absorción en las cerámicas 2 y 4 , siendo el hidrofugante $\mathrm{H} 4$ de componente siloxanos oligoméricos el que produce mayor reducción en las cerámicas 1 y 3.

La cerámica 1 (con coeficiente de absorción de 20,08 \%) hidrofugada con cualquier tipo de producto hidrofugante (excepto el H1 de componente siliconatos) es la que presenta mayor reducción de su tasa de absorción inicial.

\section{Nivel de agua superficial}

Todos los hidrofugantes reducen el nivel de agua superficial de la cerámica respecto de la cerámica sin hidrofugar $(\mathrm{H} 0)$. Para poder determinar el producto hidrofugante que produce mayor reducción de del nivel de agua superficial, se cuantifica para cada tiempo ensayado, la reducción que produce cada producto en cada tiempo respecto de la cerámica sin hidrofugar (H0). Para cada tiempo se obtiene el porcentaje ( $\%$ ) de reducción respecto a la cerámica sin tratar. De todos los porcentajes obtenidos para cada tiempo, se obtiene el porcentaje de reducción total, aplicando la media aritmética a los porcentajes obtenidos para cada tiempo ensayado. En la tabla IV se presenta el porcentaje de reducción del nivel de agua superficial respecto a la cerámica sin tratar, que produce cada hidrofugante en cada tipo de cerámica ensayada (signo + aumento; signo - disminución).

Se observa en la tabla IV que es el hidrofugante H1 de componente siliconatos es el que produce mayor reducción del nivel de agua superficial en las cerámicas 1, 3 y 4, siendo el hidrofugante $\mathrm{H} 5$ de componente resina fluorada y el H6 de componente silanos/siloxanos los que producen mayor reducción en la cerámicas 2 .

La cerámica 1 (con coeficiente de absorción de 20,08\%) hidrofugada con cualquier tipo de producto hidrofugante (excepto el H6 de componente silanos/siloxanos) es la que presenta mayor reducción de su nivel de agua superficial.

\section{CONCLUSIONES}

Todos los productos hidrofugantes reducen los parámetros de tasa de absorción inicial y nivel de agua superficial de la cerámica porosa cara vista, respecto de la cerámica sin hidrofugar (H0). La variación de dichos parámetros está determinada por el tipo de hidrofugante, reduciendo en todos los casos la tasa de absorción inicial más de un $84,37 \%$ y el nivel de agua superficial en más de un 52,55\%.
La cerámica 1, con el mayor coeficiente de absorción de las cerámicas ensayadas $(20,08 \%)$ es la que presenta la mayor reducción de la tasa de absorción inicial y del nivel de aguas superficial.

Respecto al producto hidrofugante y según los resultados obtenidos, nos permite sugerir que para la hidrofugación de piezas de cerámica porosa cara vista de similares características a la analizada en este articulo, es recomendable la elección del hidrofugante $\mathrm{H} 1$ de componente siliconatos, ya que, en general, corresponde al producto que produce la mayor reducción de la tasa de absorción y del nivel de agua superficial en todos los tipos de cerámica ensayados.

\section{REFERENCIAS}

1. A. García Santos: "Los Plásticos en la Construcción I y II". Madrid. Cuadernos del Instituto Juan Herrera, Escuela Técnica Superior de Arquitectura, pp 27-42. (2001)

2. J. A. Coronado Martín: "Influencia de los productos hidrofugantes en las propiedades superficiales de las cerámicas porosas utilizadas en cerramientos exteriores cara vista". Tesis doctoral. Departamento de Construcción y Tecnología Arquitectónicas. E.T.S. Arquiitectura. Universidad Politécnica de Madrid. 2012.

3. E. Pérez Monserrat, M. Gómez Heras, M. Álvarez de Buergo, R. Fort González: "Polímeros sintéticos para la conservación de materiales pétreos". Ciencia, Tecnología y Sociedad para una conservación sostenible del patrimonio pétreo, pp. 71-83". Dpto. Publicaciones Universidad Popular José Hierro, San Sebastián de los Reyes. 71-82. ISBN: 84-9571041-2. (2007).

4. J. A. Coronado Martín, A. García Santos: "La influencia de los productos hidrofugantes en las modificaciones cromáticas del ladrillo cerámico". Materiales de construcción. ISSN 0465-2746, vol. 61. N 304. Págs. 597-611. Octubre/noviembre/diciembre 2011. CSIC. (2011).

5. Wacker-chemie Gmbh: "Procedimiento para hidrofugar superficies de materiales de construcción". Patente de invención. 1973.

6. J. A. Coronado Martín, A. García Santos: “The influence of water repellent products on the chromatic modifications of the ceramic brick". $37^{\circ}$ IAHS Word Congress on Housing Science. "Desing, Technology, Refurbishment and management of Buildings". Santander, España, 26-29 Octubre 2010. Code 173, pag. 109. ISBN: 978-84-693-6655-4. (2010)

7. J. A. Coronado Martín, A. García Santos, J. M. Adell Argilés: “The influence of water repellent products on the chromatic modifications of the ceramic brick". 8th International Masonry Conference. Dresde, Alemania, 4-7 Julio 2010. ISBN: 978-3-00-0313881-3. (2010)

8. Norma UNE-EN 772-11:2001 "Metodos de ensayo de piezas para fábrica de albañilería. Parte 11: Determinación de la absorción de agua por capilaridad de piezas para fábrica de albañilería, en hormigón, piedra natural y artificial, y de la tasa de absorción de agua inicial de las piezas de arcilla cocida para fábrica de albañilería". 2001.

9. A. García Santos, M. Conci Rinaudo: "Variación del color del soporte cerámico tratado con pintura antigraffiti". Mater. Construcc, vol. 55. n 278 (abril/mayo/junio 2005), CSIC, pp. 55-68. (2005).

10. A. Beltramone, A. García Cruz, A. García Santos: “Modificaciones de las propiedades superficiales en el hormigón visto, por la utilización de diferentes tipos de desencofrantes y aditivos plásticos". Mater Construcc, vol 53, no 270 (abril/mayo/junio 2003), CSIC, pp. 71.78. (2003). 